\title{
An Improved Routing Algorithm for Mobile Opportunistic Networks using a Genetic Approach
}

\author{
Abdulaziz Shehab ${ }^{1,2^{*}}$, Wafaa Shaban ${ }^{1}$ and Samir Zaid ${ }^{3}$ \\ 'Department of Information Systems, Mansoura University, Mansoura - 35516, Egypt; abdulaziz_shehab@mans. \\ edu.eg, eng_wafaa_87@yahoo.com \\ ${ }^{2}$ Department of Computer Science, College of Science and Arts, Jouf University, KSA \\ 32Department of Geology, Zagazig University, Egypt; samir_Zaid75@ yahoo.com
}

\begin{abstract}
Background/Objectives: Nowadays, Mobile Opportunistic Networks (MONs) are counted as one of the most interesting development of MANETs. In MONs, forwarding messages through mobile nodes is a big challenging research problem. Methods/Analysis: In this study, an optimization technique that based on a Genetic Algorithm (GA) is proposed. It is oriented towards solving most issues faced in Delay/Disruption Tolerant Network (DTN) routing such as there is no end to end connectivity between nodes, long delay for transmission message in path, congestion during sending the message and more. Findings: The proposed technique outperforms the other state-of-art methods regarding to delivery ratio, message transmission delay and message overhead ratio. Application/Improvements: Compared to the state-of art methods, the results show that our proposed algorithm has a better performance in terms of message delivery ratio and message transmission delay, and message overhead ratio.
\end{abstract}

Keywords: Delay/Disruption Tolerant Network (DTN), Genetic, Opportunistic Networks, Optimization, Routing, MANETs

\section{Introduction}

Mobile Opportunistic Network (MON) is a category of ad hoc network, its sensors are either mobile nodes (mobile devices, cell phone) or fixed nodes (that is placed in location such as the affected area). Suddenly, if any disaster happens all over the world, the infrastructure communication is sustainable to be destroyed or fall down. Since the primary requirement during the disaster is the effective communication, message of details about the situation should be transferred between the source and destination. Delay/Disruption Tolerant Network (DTN) is required for supporting such communication between nodes. It is designed especially for supporting the distribution or less efficient of delivering message. DTN based on store and forward mechanism where the node moves/forward message stored in their memory once an opportunity appeared. By the way, the $\mathrm{MON}$ is a type of $\mathrm{DTN}^{1-3}$. Nodes have not any knowledge about either the time they will contact neither another node nor which node it will be in the contact list. Routing between sender and destination of message are built dynamically and, in that communication, any possible node can opportunistically be used as next hop. Consequently, the message will be closer to the final destination. Dependently, forwarding data in MONs is a big challenging research area ${ }^{4}$. Inside MONs, mobile nodes could communicate with each other regardless of the existence or absence of the end to end path. The next hop could be any possible node able to forward the message towards to the final destination ${ }^{5}$.

In realistic disaster while the network connectivity is intermittent and infrastructure damaged, MONs help transfer messages (using mobile devices) between the people in disaster area and destination. The destination is gathering all the information relative to the disaster carried through the people around the disaster area. The gathering process is done by sending text, photos, audio,

${ }^{*}$ Author for correspondence 
and video messages. Network topology is formed using Mobile devices which is the main core of the communication platform $\frac{3,6}{3}$.

On the other side, routing such information of the disaster must be done in real time with a high accuracy as much as possible. Routing is used to send the message between nodes towards destination. It comprises of two main components: 1 . a way discovery to destination, and 2. determination of the next hop for helpers. Therefore, the message will be buffered for a long time waiting for opportunity to route the data closer to destination.

Routing in DTN depends on the selection of optimized way for sending the message between sender and destination. The major objective of selection is the message delivery ratio. There are many different routing algorithms appeared in research area to improve the optimization. We will present some of them and comparing with our proposed algorithm ${ }^{7}$. Epidemic routing protocol $^{8}$ is depending on extremely basic idea of repeating every message stored in a node to all nodes it the contact area to ensure that the destination will receive this message, but it produces congestion and need huge bandwidth and buffer. In the other side this protocol has a higher probability for deliver message. Prophet routing protocol $^{9}$ uses an algorithm based on encounters to determine how likely each node to deliver message to destination is. Node stored probability and exchanged with other nodes when they meet. Messages forwarded only to another node that have higher delivery probability. Even if this protocol improves the performance of network but it acts like Epidemic. Max Prop routing protocol ${ }^{10}$ is a flooding-based routing which mean that there is a multiple message waiting for opportunity to send to the destination, Max Prop determining which messages should be transmitted first and which messages should be dropped first. SAW ${ }^{11}$ splashing pattern by restricting the quantity of message duplicates sent.

In this study, a proposed routing algorithm based on GA is presented to fix some issues that face other state-ofart routing algorithms. Our proposed technique depends on a GA to produce high degree of optimization. GA is a collection of candidate solutions to an optimization problem is evolved towards better solutions. The algorithm helps to define the path which the message should take if it will be transferred to the neighbor node. Using the benefit of GA with our proposed algorithm for finding the optimal path for sending the message from source to destination without delay or loss through the network.
The performance of our proposed algorithm outperforms other state-of-art Techniques (Epidemic, SAW and Max Prop routing algorithms) in terms of delivery ratio, message transmission delay and message overhead ratio. Since we are looking for the optimization, our proposed algorithm, in comparison to other routing algorithms, has the best performance in message delivery ratio and message transmission delay. In message overhead ratio our proposed algorithm has effective performance than others state-of-art routing algorithms.

The rest of the study is organized as follow: Section 2 provide a brief description of related works. Section 3 presents the proposed algorithm. Section 4 presents the simulation results. Finally, section 5 concludes the conclusion and directions for the future work.

\section{Literature Survey}

Last few years, many research studies $\frac{1-3,5,6,10-19}{10}$ on MONs have been published. However, most of these studies suffers from high network congestion and have a high requirement of network resources. A general overview of different simulators that are used in wireless and mobile network and a description of their related models could be found in ${ }^{14,20}$.

In $n^{\frac{13}{3}}$ proposed a viable message transmission technique by DTN based multi-hop network to avoid the failure of network during the disaster, the nodes send the message by multi hop technique between nodes. They support also vehicle-to-walker communication using smart devices. This strategy improves the transmission of message during the emergency cases. $\mathrm{In}^{\frac{16}{6}}$ designed frame work for mobile agent that helps any node inside the network to know the distance of other nodes. In other words, the proposed mechanism would help a node to know the approximate location related of other nodes in the system once the location information known to the destination; query response mechanism can be implemented using Geographic Routing. In $^{21}$ also work on the same point where they use mobile agents that hold information about different cases of other nodes and send it using Geographic routing.

$\mathrm{In}^{15}$ discuss how to design opportunistic routing protocols for underwater sensor networks using blocks, candidate set selection and candidate coordination procedures. Another effort focuses on existing WSN simulation frameworks is proposed by ${ }^{12}$. Their proposal could be integrated with real-time hardware prototypes. 
These simulation frameworks are analyzed and compared in order to determine a suitable simulation environment that supports specific software packages.

$\mathrm{In}^{19}$ discuss the using of GPS in opportunistic network during emergency cases. They describe the results by tracing the emergency cases in fire department for one year. In $\frac{22}{}$ reviews the different routing protocols, simulators, network topologies and mobility models that used during disaster cases to help the developers to increase the performance of this types of network. $\operatorname{In}^{6}$ develop routing algorithm for MONs to handle and response the disasters by studying the routing problem such as delivery ratio of messages, the overhead ratio of messages, and the average delay of messages in MONs. $\mathrm{In}^{23}$ provides evacuation support to people in emergences by using Emergency Support System (ESS) that uses opportunistic contacts between wireless communication devices carried by people to send information about the current situation, it provide emergency message in order to show the evacuations safety to the exit.

$\mathrm{In}^{Z}$ analysis the performance of routing protocols in opportunistic network during the emergency cases. They used number of people to show how they act during this disaster using the performance routing protocols. $\mathrm{In}^{18}$ shows a performance evaluation of joint routing in MONs using the devices carried by people by specific simulator called ONE.

\section{The Proposed Algorithm}

MON's sensors are either mobile nodes (mobile devices, cell phone) or fixed nodes (that is placed in location such as the affected area). Each oppnet starts from seed node (set of nodes that are together at the time of oppnet development). It can be a single node. The seed grows into a larger network by sending invitations to other nodes such as foreign devices, node clusters, or others which are able to contact. These nodes called Helpers. Any helpers become a member of oppnet have the responsibility to invite other nodes. Helpers of oppnet together collaborate on achieving the opportunistic network goals. Helpers are dynamically expanded to execute all kinds of tasks. During the disaster, seed oppnet starts to invite other nodes to expand the network. By integrating helpers; a seed oppnet grows into an expanded oppnet. Figure 1 shows a drawing for a presumptive MON.

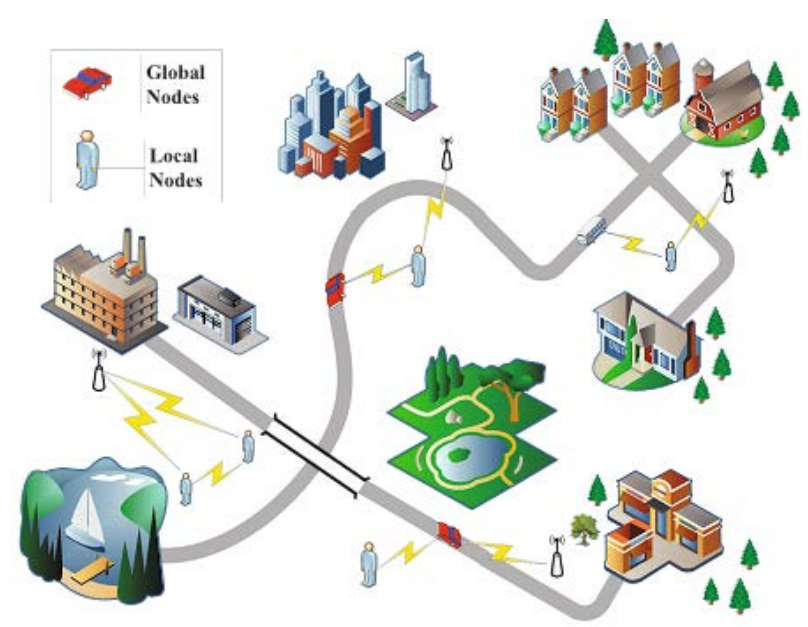

Figure 1. A presumptive mobile opportunistic network ${ }^{24}$.

In order to achieve the goals of MONs, it was necessary to test all the available protocols and enhance them to interact with the disasters efficiently. During the disaster, the network topology is built according to the nodes and the helpers that has been invited which is allocated in the area. Such nodes and helpers are working together to perform accurate data about the situation to send it to the destination. Figure 2 shows a disaster scenario. For example, if there is fire appears in one of building, one of the helpers sampling rates for temperature and wind direction get alarmed. Other helper looks at a public area scene with many objects. The message is passed to another helper that analyzes it and recognizes one of the objects as a mobile device. This mobile device looks for another helper to send the collected data.

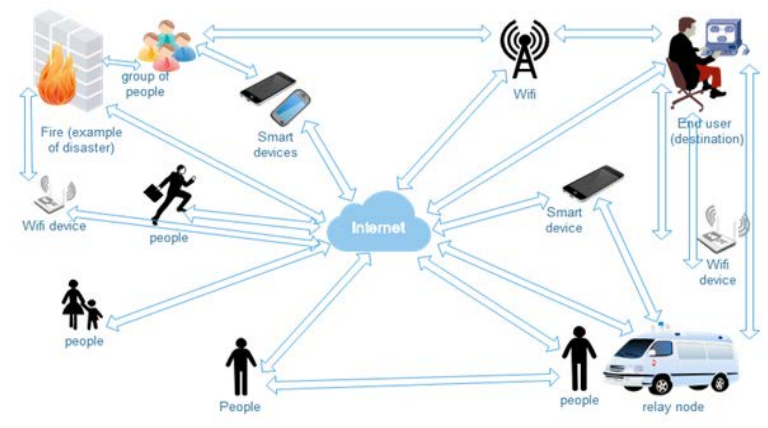

Figure 2. A depiction for a disaster scenario.

Sequentially, the message is routed from a person to another through their mobile devices until reach to the destination which called the fire station and ambulance takes its procedures. 
In DTNs, routing can be modeled as an optimization problem with the goal of finding the optimal path that will be chosen from multi-paths between source and destination node. A GA is an optimization approach based on a population of chromosomes. Algorithm 1 summarized the steps of our proposed technique with a population size $=100$.

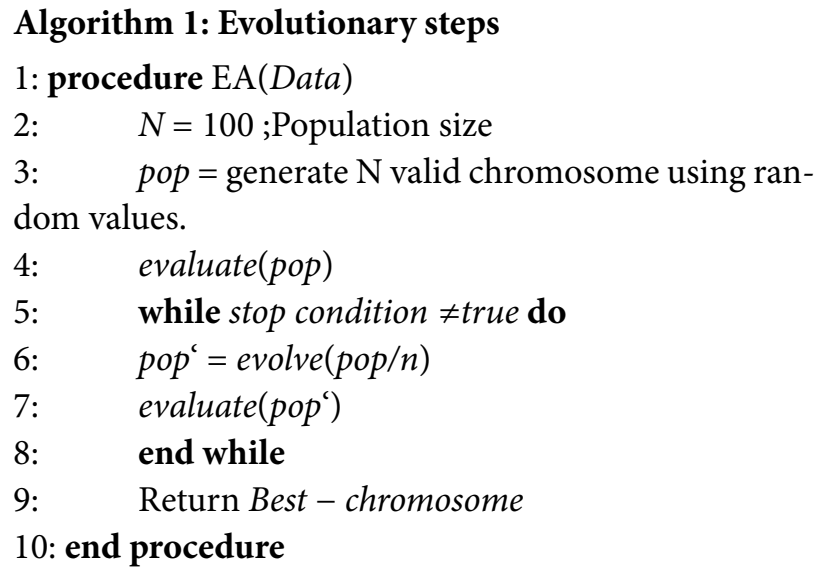

Initially the valid population of chromosomes is randomly generated. Then, to choose the best chromosomes for the next generation, these chromosomes are passed to a crossover process, followed by a mutation process. Evolutionary mutual information is illustrated in algorithm 2 which evaluate the population through a fitness function. Selection, crossover, mutation, and generation processes are repeated until a stopping criterion is satisfied. The stopping condition is either finding the optimal solution (Fitness $=100 \%$ ) or reaching repetition count.

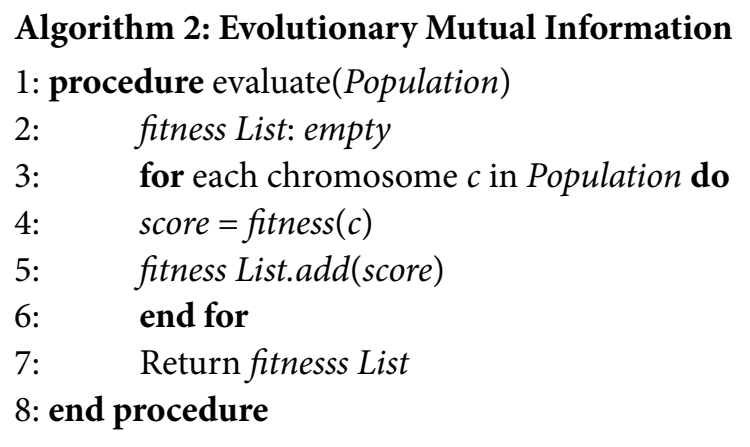

In our model, the nodes inside the MON are represented as gene. Algorithm 3 presents the details of crossover operator and mutation operator. The best solutions in general Pop are assigned to Pop1. Thereafter, the crossover operator is performed through selecting two random solutions $\mathrm{A}$ and $\mathrm{B}$ from Pop. The new generated solutions $\mathrm{C}$ and $\mathrm{D}$ are assigned to Pop2. Afterwards, the mutation is done by mutating every bit in solution $\mathrm{c}$ in Pop2 according to an elitism ratio er. The new generated solution c' with the original c solution in Pop2. Finally both Pop1 and Pop2 are merged into Pop'.

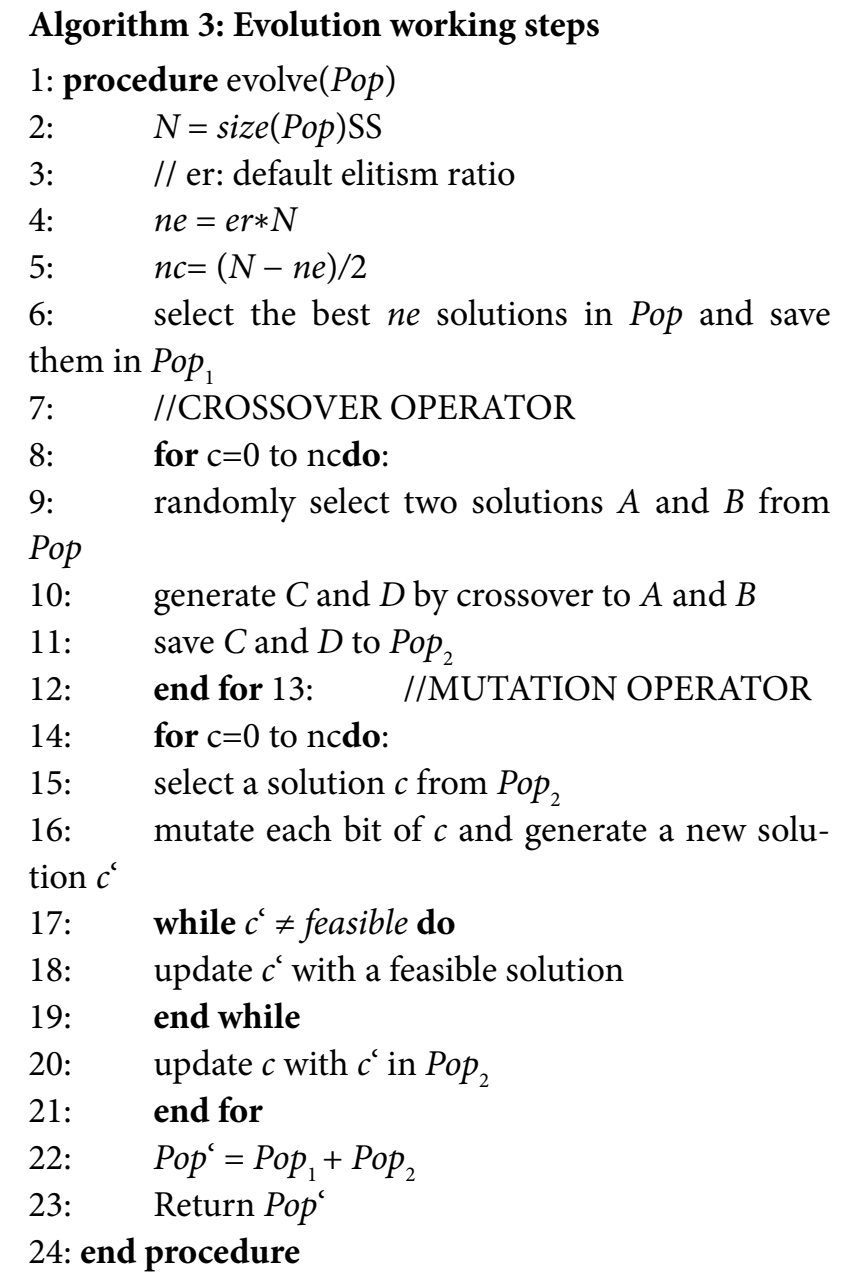

\section{Simulation Results}

\subsection{Experiment and Parameters}

Simulation experiments are executed using Net Beans IDE over the Opportunistic Network Environment (ONE) simulator $\frac{24,25}{2}$. Three main state-of-art methods namely Epidemic, Maypop, and SAW are used in the experiment beside our proposed algorithm. The total number of nodes in the network is 124 . The whole simu- 
lation time is 45,000 seconds and the initialization time is 1800 seconds. Table 1 presents a description for the simulation parameters used in the experiment.

Table 1. Simulation parameters

\begin{tabular}{ll}
\hline Parameter & Value \\
\hline Simulation area & $4,500 \times 3,400 \mathrm{~m}$ \\
Node bandwidth & $2 \mathrm{Mbps}$ \\
Node velocity & $0.5 \sim 1.5 \mathrm{~m} / \mathrm{s}$ \\
Node transmit range & $20 \mathrm{~m}$ \\
Message size & $0.5 \sim 1.5 \mathrm{MB}$ \\
Message TTL & $3,600 \mathrm{~s}$ \\
Initial energy of node & $10,000 \mathrm{~mA}^{\star} \mathrm{h}$ \\
Energy expenditure per & $10 \mathrm{~mA}^{\star} \mathrm{h}$ \\
scan & \\
Transmit energy & $20 \mathrm{~mA}^{\star} \mathrm{h}$ \\
expenditure per sec & \\
\hline
\end{tabular}

\subsection{A Comparative Study}

Initially, Epidemic routing protocol depends extremely on the idea of repeating every message stored in a node to all nodes found in the contact area to ensure that the destination will receive the disaster message. Therefore, it generates a high congestion and need huge bandwidth and buffer. However, it has a higher probability for message delivery. Max Prop protocol is a flooding-based routing protocol which means that there is a multiple message waiting for opportunity to send to the destination. Max Prop decides which messages should be transmitted and which messages should be dropped out. Finally, SAW protocol makes a restriction on the quantity of message duplicates sent. Figure 3-5 are showing the results of comparing our proposed to the other state-of-art methods. Three main factors are used in the comparison: message delivery ratio, message transmission delay, and message overhead ratio. As shown in Figure 3, the proposed algorithm outperforms the others in terms of delivery ratio. When the buffer size is between 10 to $30 \mathrm{MB}$, the delivery ratio is almost equal for the four algorithms. However, by increasing the buffer size, the delivery ratio for our proposed algorithm is increased significantly compared to other algorithms. This is due to usage of genetic which has the advantage of predicting message paths in more accurate ways. As shown in Figure 4, the proposed algorithm has the minimal message transmission delay than the others. It grows slowly by increasing the buffer size.
The proposed routing algorithm achieves the optimization through selecting the best path to send the message without losing time. Both Epidemic and Max Prop record the worst message delay while SAW has a second better performance after our proposed algorithm.

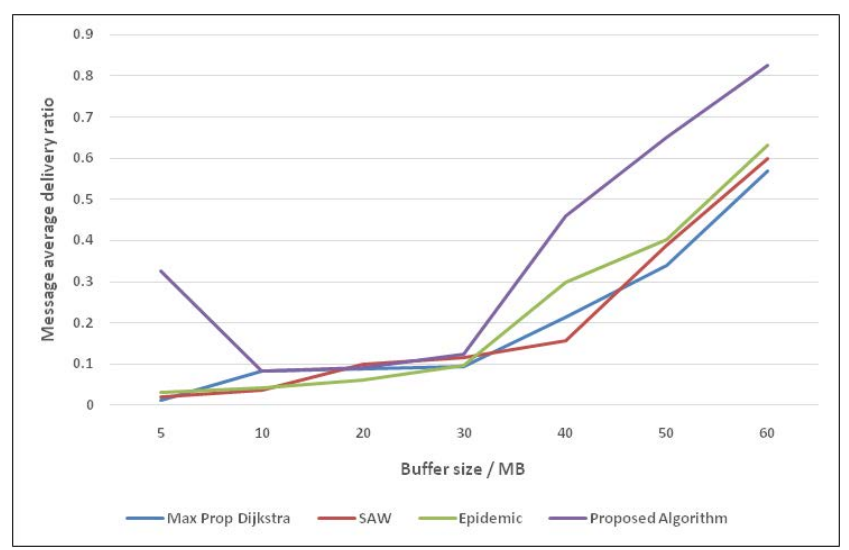

Figure 3. Delivery ratio vs. buffer size.

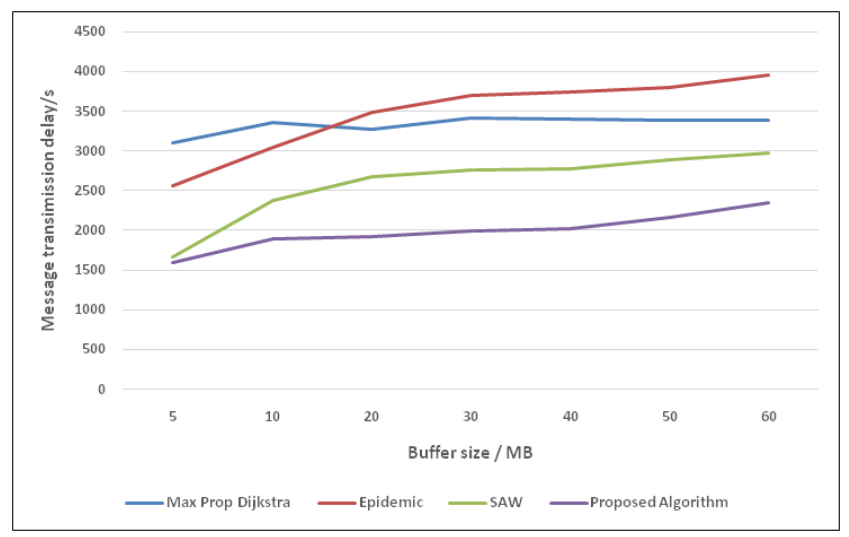

Figure 4. Delay time vs. buffer size.

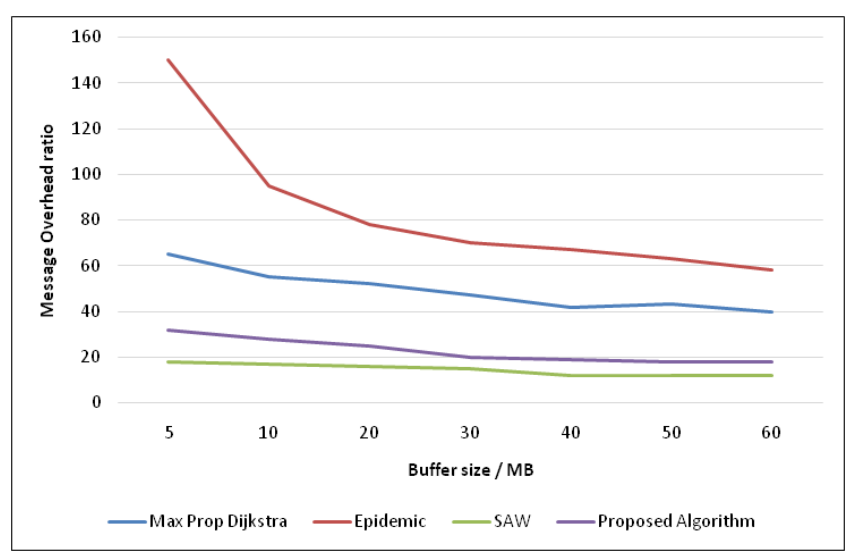

Figure 5. Overhead ratio vs. buffer size.

As illustrated in Figure 5, the SAW achieved the best performance in terms of message overhead ratio. 
However, SAW works on forwarding the message to the destination node instantly in contrary to Epidemic, Max Prop, and the proposed algorithm which they have several copies to send. Despite of this advantage to SAW, there is a minor improvement compared to the proposed algorithm. As depicted in Figure 5, the proposed algorithm is dramatically outperformed both Epidemic and MaxProp. Moreover, we plan to refine the proposed algorithm to be suitable for more challenging environments, such as throw box-equipped DTNs ${ }^{26}$, vehicular DTNs ${ }^{27}$.

\section{Conclusion and Future Work}

In order to increase message delivery ratio and reduce overhead in MONs, routes must be developed powerfully. However, most of the available classic routing algorithms lack some issues in terms of message delivery ratio, overhead ratio, and delay time. In this study, a novel routing algorithm that based on a genetic approach is proposed. The simulation results shows that proposed algorithm outperform all of state-of-art routing algorithms for DTNs in terms of both message delivery ratio and delay time. The proposed algorithm achieves the second best message overhead ratio behind SAW because it just forwards message to the destination node in the wait phase. In the future, we look to achieve the best performance in message overhead ratio by improving the optimization technique of the proposed algorithm.

\section{References}

1. Dutt I. Issues in delay tolerant networks: A comparative study, International Journal of Advanced Research in Computer Science and Software Engineering. 2015; 5(6):534-42.

2. Routing Issues and Performance of Different Opportunistic Routing Protocols in Delay Tolerant Network. Date accessed: 2016. https://www.semanticscholar.org/paper/ Routing-Issues-\%26-Performance-Of-Different-RoutingUpadhyay-Mishra/6cd28d14c7b38d030b6b4d95fd65da16 b55e57c6.

3. Kitada S, Sato G, Shibata Y. A DTN-Based Multi-hop Network for Disaster Information Transmission. Smart Sensors Networks; 2017. p. 53-66. https://doi.org/10.1016/ B978-0-12-809859-2.00004-8. PMid: 28593583.

4. A 3D location-based energy aware routing protocol in delay tolerant networks. Date accessed: 24/08/2014. https:// ieeexplore.ieee.org/document/6945738.
5. Ali AHA. Optimum bridge site selection using network analysis tool (Al Muwaffaqiyah Bridge in Iraq as A case Study), Journal of University of Babylon for Engineering Sciences. 2018; 26(2):198. Muwaffaqiyah 213. https://doi. org/10.29196/jub.v26i2.385.

6. Wang X. A social activity and physical contact-based routing algorithm in mobile opportunistic networks for emergency response to sudden disasters, Enterprise Information Systems. 2017; 11(5):597-626. https://doi.org /10.1080/17517575.2015.1067840.

7. MartíN-Campillo A. Evaluating opportunistic networks in disaster scenarios, Journal of Network and Computer Applications. 2013; 36(2):870-80. https://doi.org/10.1016/j. jnca.2012.11.001.

8. Survey on routing algorithms in opportunistic networks. Date accessed: 04/01/2013. https://ieeexplore.ieee.org/ abstract/document/6466129.

9. Probabilistic routing in intermittently connected networks. Date accessed: 2003. http://citeseerx.ist.psu.edu/viewdoc/ download?doi=10.1.1.59.6027\&rep=rep1\&type=pdf.

10. Harrati Y, Abdali A. Max Hop Count: DTN congestion control algorithm under Max Prop routing, International Journal of Computer Science and Network Security. 2017; 17(5):206-14.

11. Guo H. A location aided controlled spraying routing algorithm for Delay Tolerant Networks, Ad Hoc Networks. 2017; 66:16-25. https://doi.org/10.1016/j.adhoc.2017.08.005.

12. Rajaram ML. Wireless sensor network simulation frameworks: A tutorial review: MATLAB/Simulink bests the rest, IEEE Consumer Electronics Magazine. 2016; 5(2):63-69. https://doi.org/10.1109/MCE.2016.2519051.

13. Kitada S, Sato G, Shibata Y. Performance Evaluation of a DTN Based Multi-hop Network for Disaster Information Transmission by Smart Devices. International Conference on Broadband and Wireless Computing, Communication and Applications; 2016. p. 601-11. https://doi. org/10.1007/978-3-319-49106-6_59.

14. Georgioski I, Trenchevska H. Wireless sensor networks simulators-computer simulation tools. On Applied Internet and Information Technologies; 2016. p. 199. https://doi. org/10.20544/AIIT2016.24.

15. Coutinho RW. Design guidelines for opportunistic routing in underwater networks, IEEE Communications Magazine. 2016; 54(2):40-48. https://doi.org/10.1109/ MCOM.2016.7402259.

16. Bandyopadhyay S, Mukherjee A. Tracking user-movement in opportunistic networks to support distributed query-response during disaster management, Procedia Engineering. 2016; 159:82-88. https://doi.org/10.1016/j. proeng.2016.08.068. 
17. Saha S. Designing delay constrained hybrid ad hoc network infrastructure for post-disaster communication, Ad Hoc Networks. 2015; 25:406-29. https://doi.org/10.1016/j. adhoc.2014.08.009.

18. Roohinaz M, Shah MB. Performance evaluation of joint routing and scheduling in opportunistic sensor networks, International Journal of Computer Applications. 2015; 122(20):18-23. https://doi.org/10.5120/21816-5144.

19. Sergio Cabrero, Roberto García, Xabiel G. Pañeda, David Melendi. Understanding opportunistic networking for emergency services: Analysis of one year of GPS traces. CHANTS ' 15 Proceedings of the 10th ACM MobiCom Workshop on Challenged Networks. ACM New York, NY, USA; 2015. p. 31-36. Date accessed: 2015. https://dl.acm. org/citation.cfm?id=2799371.2799381.

20. Opportunistic networks: The concept and research. In Challenges in Privacy and Security. Date accessed: 01/2007. https://www.researchgate.net/publication/237295326_ Opportunistic_networks_the_concept_and_research_ challenges_in_privacy_and_security.

21. Choudhury RR, Paul K, Bandyopadhyay S. MARP: A multi-agent routing protocol for mobile wireless ad hoc networks, Autonomous Agents and Multi-Agent Systems. 2004; 8(1):47-68. https://doi. org/10.1023/B:AGNT.0000009410.57024.9a.
22. Rosas E. Survey on simulation for mobile Ad-Hoc communication for disaster scenarios, Journal of Computer Science and Technology. 2016; 31(2):326-49. https://doi. org/10.1007/s11390-016-1630-x.

23. Gorbil G. Opportunistic communications for emergency support, Procedia Computer Science. 2011; 5:39-47. https://doi.org/10.1016/j.procs.2011.07.008.

24. Simulation based Performance Comparison and Analysis regarding Static and Mobile Throwboxes impact on Network Performance in Delay Tolerant Networks (DTNs) using ONE Simulator. Date accessed: 2018. https://iopscience.iop.org/article/10.1088/1742-6596/1142/1/012015.

25. Han W, Xiao Y. Deploying Throwboxes to enhance fault-tolerance performance in delay tolerant networks, Wireless Personal Communications. 2018; 99(3):1247-78. https://doi.org/10.1007/s11277-017-5183-2.

26. A Distance-Based Advertisement-Delivery Method for Vehicular DTN. Date accessed: 03/2019. https:/www. researchgate.net/publication/331767598_A_DistanceBased_Advertisement-Delivery_Method_for_Vehicular_ DTN.

27. Yuan P, Wang C. OPPO: An optimal copy allocation scheme in mobile opportunistic networks, Peer-to-Peer Networking and Applications. 2018; 11(1):102-09. https:// doi.org/10.1007/s12083-016-0472-1. 